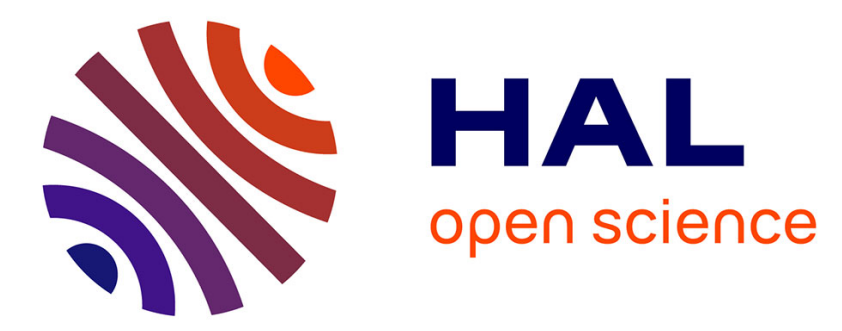

\title{
Reliable H-infinity Filtering for Discrete Time-Delay Markovian Jump Systems with Partly Unknown Transition Probabilities
}

Yisha Liu, Zidong Wang, Wei Wang

\section{- To cite this version:}

Yisha Liu, Zidong Wang, Wei Wang. Reliable H-infinity Filtering for Discrete Time-Delay Markovian Jump Systems with Partly Unknown Transition Probabilities. International Journal of Adaptive Control and Signal Processing, 2011, 25 (6), pp.554. 10.1002/acs.1231 . hal-00614057

\section{HAL Id: hal-00614057 https://hal.science/hal-00614057}

Submitted on 9 Aug 2011

HAL is a multi-disciplinary open access archive for the deposit and dissemination of scientific research documents, whether they are published or not. The documents may come from teaching and research institutions in France or abroad, or from public or private research centers.
L'archive ouverte pluridisciplinaire $\mathbf{H A L}$, est destinée au dépôt et à la diffusion de documents scientifiques de niveau recherche, publiés ou non, émanant des établissements d'enseignement et de recherche français ou étrangers, des laboratoires publics ou privés. 


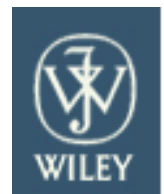

\section{Reliable H-infinity Filtering for Discrete Time-Delay Markovian Jump Systems with Partly Unknown Transition Probabilities}

\begin{tabular}{|r|l|}
\hline Journal: & International Journal of Adaptive Control and Signal Processing \\
\hline Manuscript ID: & ACSP-10-0174.R1 \\
\hline Wiley - Manuscript type: & Research Article \\
\hline Complete List of Authors: & $\begin{array}{l}\text { Liu, Yisha; Dalian University of Technology, Research Centre of } \\
\text { Information and Control } \\
\text { Wang, Zidong; Brunel University, Information Systems and } \\
\text { Computing } \\
\text { Wang, Wei; Dalian University of Technology, Research center of } \\
\text { Information and Control }\end{array}$ \\
\hline Keywords: & $\begin{array}{l}\text { H-infinity filtering, Reliable filtering, Sensor failure, Markovian jump } \\
\text { systems, Partly unknown transition probabilities }\end{array}$ \\
\hline
\end{tabular}

\section{SCHOLARONE $^{\text {M }}$ \\ Manuscripts}




\section{Explanation of this revision}

Paper number: ACSP-10-0174

First of all, the authors would like to express their sincere thanks to the Editor and the anonymous reviewer for their helpful comments and suggestions. The explanation of the modifications as well as corrections in this revision can be arranged as follows (comment numbers are in 1:1 correspondence with the reviewers' comments).

\section{Reply to Dr. Andrea Lecchini-Visintini}

Thank you very much for your time and efforts in coordinating the review process. Your comprehensive summary of the review reports is much appreciated. In this revision, all the comments from the reviewers have been seriously taken into account and thoroughly implemented.

\section{Reply to Reviewer No. 1}

Thanks to this reviewer for the encouraging comments.

\section{1) Page 2, the 3rd paragraph}

In this revision, the following comment has been added to point out the advantage and significance comparing with existing papers that addressed the stability of Markovian jumping systems with incomplete transition probabilities:

"Comparing with [28,29], both the nonlinearities and time-varying delays are considered for Markovian jumping systems and therefore the model in this paper is more general. In addition, the reliability issue is studied and a reliable filter is designed in the presence of possible sensor failures. The main contributions are as follows. 1) The transition probabilities of the jumping process are assumed to be partly unknown. The developed results are more general since they can be applied to Markovian jumping systems with completely known, completely unknown and partly unknown transition probabilities. 2) The nonlinearities are introduced as exogenous nonlinear disturbances which are described by statistical means. 3) The sensor failures are described by a variable taking values in some interval. Note that such a description is more practical than the conventional outage case. 4) By using a novel Lyapunov-Krasovskii functional and delay-partitioning technique, delay-dependent sufficient conditions are obtained under which the filtering error system is asymptotically mean-square stable with an $H_{\infty}$ disturbance attenuation level $\gamma$. 5) An extra variable is introduced to realize the decoupling between the Lyapunov matrices and the filtering error system matrices in order to reduce the conservatism." 
2) Page 2, the 3rd paragraph

In this revision, the following comment has been added to further strengthen the motivation for this paper.

"Comparing with [28,29], both the nonlinearities and time-varying delays are considered for Markovian jumping systems and therefore the model in this paper is more general. In addition, the reliability issue is studied and a reliable filter is designed in the presence of possible sensor failures."

3) Page 16, Conclusion

In this revision, the following comment has been added in the conclusion part on the future research topics:

"The future research topics would include the extension of the main results developed in this paper to more general complex systems such as networked systems with random packet losses, general stochastic systems, polynomial nonlinear systems and functional differential equations of the neutral type."

4) Throughout the paper, block-diagonal matrices have been used to make the big matrices in a more compact format and similar LMIs have been rewritten in a uniform expression, see e.g. inequality (40) on page 13.

5) The references

In this revision, the following papers have been added as references.

Z. Wu, H. Su and J. Chu, $H_{\infty}$ filtering for singular systems with time-varying delay, International Journal of Robust and Nonlinear Control, vol. 20, no. 11, pp. 12691284, 2010.

Z. Wu, H. Su and J. Chu, $H_{\infty}$ filtering for singular Markovian jump systems with time delay, International Journal of Robust and Nonlinear Control, vol. 20, no. 8, pp. 939-957, 2010.

\section{Reply to Reviewer No. 2}

Thanks to this reviewer for the very positive comments.

\section{(1) Page 2, the 3rd paragraph}

In this revision, the main contributions have been explicitly stated as follows:

"The main contributions are as follows. 1) The transition probabilities of the jumping process are assumed to be partly unknown. The developed results are more general since they can be applied to Markovian jumping systems with completely known, completely unknown and partly unknown transition probabilities. 2) The nonlinearities 
are introduced as exogenous nonlinear disturbances which are described by statistical means. 3) The sensor failures are described by a variable taking values in some interval. Note that such a description is more practical than the conventional outage case. 4) By using a novel Lyapunov-Krasovskii functional and delay-partitioning technique, delay-dependent sufficient conditions are obtained under which the filtering error system is asymptotically mean-square stable with an $H_{\infty}$ disturbance attenuation level $\gamma$. 5) An extra variable is introduced to realize the decoupling between the Lyapunov matrices and the filtering error system matrices in order to reduce the conservatism."

(2) Page 10, Remark 2

In this revision, the following comment has been added in Remark 2 on the partitioning number $m$ and the computational problem:

"If $m$ increases, the dimensions of the LMIs will become larger and the computational burden will increase. Therefore, the partitioning number $m$ should be chosen properly."

(3) Page 4, Remark 1

In this revision, the following remark has been added on the statistically described nonlinearities:

"Remark 1: The nonlinearities in (2) have been described by statistical means in [36] and such description could cover several classes of well-studied nonlinear systems, for example, the nonlinear systems with random sequences whose powers depend on the sector-bounded nonlinear function of the state. Note that the time-delay term is involved in (2), and therefore the model studied in this paper is more general than the corresponding ones in [36]."

(4) Page 16, Conclusion

In this revision, the following comment has been added in the conclusion part on the future research topics:

"The future research topics would include the extension of the main results developed in this paper to more general complex systems such as networked systems with random packet losses, general stochastic systems, polynomial nonlinear systems and functional differential equations of the neutral type."

(5) The references

In this revision, the following papers have been added as references.

H. Gao, Y. Zhao, J. Lam, and K. Chen, $H_{\infty}$ fuzzy filtering of nonlinear systems with intermittent measurements, IEEE Transactions on Fuzzy Systems, vol. 17, no. 2, pp. 291-300, 2009. 
X. Meng, J. Lamb, B. Du and H. Gao, A delay-partitioning approach to the stability analysis of discrete-time systems, Automatica, vol. 46, pp. 610-614, 2010. 


\title{
Reliable $H_{\infty}$ Filtering for Discrete Time-Delay Markovian Jump Systems with Partly Unknown Transition Probabilities
}

\author{
Yisha Liu, Zidong Wang and Wei Wang
}

\begin{abstract}
In this paper, the reliable $H_{\infty}$ filtering problem is studied for a class of discrete nonlinear Markovian jump systems with sensor failures and time delays. The transition probabilities of the jumping process are assumed to be partly unknown. The failures of sensors are quantified by a variable taking values in a given interval. The time-varying delay is unknown with given lower and upper bounds. The purpose of the addressed reliable $H_{\infty}$ filtering problem is to design a mode-dependent filter such that the filtering error dynamics is asymptotically mean-square stable and also achieves a prescribed $H_{\infty}$ performance level. By using a new Lyapunov-Krasovskii functional and delay-partitioning technique, sufficient delay-dependent conditions for the existence of such a filter are obtained. The filter gains are characterized in terms of the solution to a convex optimization problem that can be easily solved by using the semi-definite programme method. A numerical example is provided to demonstrate the effectiveness of the proposed design approach.
\end{abstract}

\section{Keywords}

$H_{\infty}$ filtering; Reliable filtering; Sensor failure; Markovian jump systems; Partly unknown transition probabilities; Delay partitioning.

\section{INTRODUCTION}

In the past few years, Markovian jumping systems have received a great deal of attention since this class of hybrid systems can model different types of dynamic systems subject to abrupt changes in their structures, e.g. failure prone manufacturing systems, power systems and economics systems. For linear Markovian jumping systems, many important issues have been studied extensively such as stability and stabilization $[8,17,25]$, control synthesis $[1,4,28]$ and filter design $[21,29,37]$. Recently, much effort has been paid to the nonlinear Markovian jumping systems and some results have been reported [5,23,30], where the nonlinearities are often introduced in the form of exogenous nonlinear disturbances which may result from the linearization process of an originally highly nonlinear plant or may be an external nonlinear input. It should be pointed out that all the results mentioned above have assumed that the transition probabilities of the Markovian jumping process are completely known. Unfortunately, this is not always the case in reality. For example, in a networked control system, the transition probabilities themselves may be uncertain or even unknown due primarily to the random nature of the network-induced phenomenon. Therefore, it is significant and challenging to study Markovian jumping systems with partly unknown transition probabilities, see $[28,29]$ for some recently available works.

In practice, time delays are frequently encountered in dynamical systems because of the finite switching speed of the amplifiers. The existence of time-delays may deteriorate the system performance and even result

Y. Liu and W. Wang are with the Research Centre of Information and Control, Dalian University of Technology, Dalian 116023, China.

Z. Wang is with the Department of Information Systems and Computing, Brunel University, Uxbridge, Middlesex, UB8 3PH, United Kingdom. (Email: Zidong. Wang@brunel.ac.uk) 
in the instability of the systems $[2,3,10,11,14,36]$. Specifically, for Markovian jumping systems with time delays, a great number of results have been reported, see e.g. [5, 6, 27, 32] and the references therein. In the early results, delay-independent approach was used to deal with time delay and such an approach can be applicable to delays of arbitrary length. Since the stability of systems is explicitly dependent on the time-delay, the delay-dependent approach has been widely exploited for Markovian jumping systems, which is generally less conservative than the delay-independent one especially in situations where delays are small $[7,13,30,37]$. Recently, the so-called delay partitioning technique has been used to address the stability analysis problem of time-delay systems, which has proven to be very effective in reducing the possible conservatism of the stability criteria $[8,12,31,35]$.

On the other hand, almost all practical control systems comprise a large number of sensors. For example, networked control systems (NCSs) have been extensively applied in many technological fields and therefore attracted an increasing research attention. NCSs typically contain numerous sensors, controllers and actuators among which signals are delivered via network communication. However, in practical applications, temporal failures may happen to actuators/sensors and, subsequently, the delivered signals may be incomplete. The occurrence of such a phenomenon may affect the expected control and estimate performance. Hence, it is of engineering significance to design reliable controllers/filters in the presence of possible actuator/sensor failures. For linear systems, the reliable design problem has been thoroughly investigated and many results have been reported, see e.g. [16,20,26]. As for the nonlinear systems, the reliable control/filtering problems with actuator or sensor failures have also attracted much research interest. For example, in [19], a Hamilton-Jacobi equation approach has been used to study the reliable control problem for affine uncertain nonlinear systems. Modified algebraic Riccati equation approach has been developed in [22] to deal with the problem of robust reliable control design for a class of nonlinear uncertain state-delayed systems. In $[9,15,18,34]$, the reliable control and filtering problems have been dealt with via the linear matrix inequality (LMI) approach. However, up to now, the reliable filtering problem for discrete-time nonlinear Markovian jump systems with partly unknown transition probabilities and time-varying delays has not been fully investigated, and this gives the motivation of our present investigation.

In this paper, we focus on the reliable $H_{\infty}$ filtering problem against sensor failures for a class of discretetime nonlinear Markovian jumping systems with time-varying delays. Comparing with [28, 29], both the nonlinearities and time-varying delays are considered for Markovian jumping systems and therefore the model in this paper is more general. In addition, the reliability issue is studied and a reliable filter is designed in the presence of possible sensor failures. The main contributions are as follows. 1) The transition probabilities of the jumping process are assumed to be partly unknown. The developed results are more general since they can be applied to Markovian jumping systems with completely known, completely unknown and partly unknown transition probabilities. 2) The nonlinearities are introduced as exogenous nonlinear disturbances which are described by statistical means. 3) The sensor failures are described by a variable taking values in some interval. Note that such a description is more practical than the conventional outage case. 4) By using a novel Lyapunov-Krasovskii functional and delay-partitioning technique, delay-dependent sufficient conditions are obtained under which the filtering error system is asymptotically mean-square stable with an $H_{\infty}$ disturbance attenuation level $\gamma .5$ ) An extra variable is introduced to realize the decoupling between the Lyapunov matrices and the filtering error system matrices in order to reduce the conservatism. Based on the decoupling idea, we design a mode-dependent reliable $H_{\infty}$ filter whose gain can be obtained by solving a set of LMIs. Finally, a simulation example is utilized to illustrate the effectiveness of the proposed approach.

Notation The following notation will be used in this paper. $\mathbb{R}^{n}$ denotes the $n$ dimensional Euclidean space. 
The notation $X \geq Y$ (respectively, $X>Y$ ), where $X$ and $Y$ are symmetric matrices, means that $X-Y$ is positive semi-definite (respectively, positive definite). $\mathbb{E}\{x\}$ stands for the expectation of the stochastic variable $x . I$ and 0 represent the identity matrix and a zero matrix with appropriate dimension, respectively. For a matrix $R, R^{T}$ represents its transpose and $\operatorname{diag}\left\{R_{1}, R_{2}, \ldots\right\}$ denotes a block diagonal matrix whose diagonal blocks are given by $R_{1}, R_{2}, \ldots$. In symmetric block matrices, the symbol $*$ is used as an ellipsis for terms induced by symmetry. Matrices, if they are not explicitly stated, are assumed to have compatible dimensions.

\section{Problem Formulation}

Consider the following discrete-time nonlinear system with Markovian jumping parameters:

$$
\begin{aligned}
x(k+1) & =A\left(r_{k}\right) x(k)+A_{d}\left(r_{k}\right) x(k-d(k))+D\left(r_{k}\right) w(k)+f\left(x(k), x(k-d(k)), r_{k}\right), \\
y(k) & =C_{1}\left(r_{k}\right) x(k)+D_{1}\left(r_{k}\right) w(k), \\
z(k) & =C\left(r_{k}\right) x(k), \\
x(k) & =\varphi(k), \quad k=-d_{M},-d_{M}+1, \ldots, 0,
\end{aligned}
$$

where $x(k) \in \mathbb{R}^{n}$ is the state; $y(k) \in \mathbb{R}^{p}$ is the measured output vector; $z(k) \in \mathbb{R}^{r}$ is the signal to be estimated; and $w(k) \in \mathbb{R}^{h}$ is the exogenous disturbance signal which is assumed to belong to $l_{2}[0 \infty)$. $d(k)$ denotes the time-varying delay with lower and upper bounds $d_{m} \leq d(k) \leq d_{M}$. Here, the lower bound of delay $d_{m}$ can always be written by $d_{m}=\tau m$, where $\tau$ and $m$ are integers. $\varphi(k)$ is the initial state of the system. $A\left(r_{k}\right), A_{d}\left(r_{k}\right), C\left(r_{k}\right), C_{1}\left(r_{k}\right), D\left(r_{k}\right)$ and $D_{1}\left(r_{k}\right)$ are matrix functions of the random jumping process $r_{k}$. $r_{k}$ is a Markov chain taking values in a finite state space $S=\{1,2, \ldots, N\}$ with transition probability matrix $\Lambda=\left(\pi_{i j}\right)_{N \times N}$ given by

$$
P_{r}\left\{r_{k+1}=j \mid r_{k}=i\right\}=\pi_{i j}, \quad \forall i, j \in S
$$

where $0 \leq \pi_{i j} \leq 1(i, j \in S)$ is the transition rate from $i$ to $j$ and $\sum_{j=1}^{N} \pi_{i j}=1, \forall i \in S$. In this paper, the transition probabilities of the jumping process are assumed to be partly accessed, i.e., some elements in matrix $\Lambda$ are unknown. For example, for system (1) with four modes, the transition probability matrix $\Lambda$ may be

$$
\Lambda=\left[\begin{array}{cccc}
\pi_{11} & ? & \pi_{13} & ? \\
? & \pi_{22} & ? & ? \\
\pi_{31} & ? & ? & \pi_{34} \\
? & \pi_{42} & ? & \pi_{44}
\end{array}\right]
$$

where "?" represents the inaccessible element. For notation clarity, for any $i \in S$, we denote that

$$
\zeta_{\kappa}^{i} \triangleq\left\{j: \pi_{i j} \text { is known }\right\}, \quad \zeta_{u \kappa}^{i} \triangleq\left\{j: \pi_{i j} \text { is unknown }\right\} .
$$

The nonlinear function $f\left(x(k), x(k-d(k)), r_{k}\right)$ consisting of $x(k)$ and $x(k-d(k))$ is bounded in a statistical sense as follows:

$$
\begin{aligned}
& \mathbb{E}\left\{f\left(x(k), x(k-d(k)), r_{k}\right) \mid x(k), x(k-d(k)), r_{k}\right\}=0, \\
& \mathbb{E}\left\{f\left(x(k), x(k-d(k)), r_{k}\right) f^{T}\left(x(k), x(k-d(k)), r_{k}\right) \mid x(k), x(k-d(k)), r_{k}\right\} \\
& =\sum_{l=1}^{q} \theta_{l}\left(r_{k}\right) \theta_{l}^{T}\left(r_{k}\right)\left(x^{T}(k) \Xi_{l}\left(r_{k}\right) x(k)+x^{T}(k-d(k)) \Pi_{l}\left(r_{k}\right) x(k-d(k))\right) \\
& \text { http://mc.manuscriptcentral.com/acsp-wiley }
\end{aligned}
$$


where $\theta_{l}\left(r_{k}\right)(l=1, \ldots, q)$ are known mode-dependent column vectors, and $\Xi_{l}\left(r_{k}\right)$ and $\Pi_{l}\left(r_{k}\right)$ are known positive-definite mode-dependent matrices.

Remark 1: The nonlinearities in (2) have been described by statistical means in [33] and such description could cover several classes of well-studied nonlinear systems, for example, the nonlinear systems with random sequences whose powers depend on the sector-bounded nonlinear function of the state. Note that the time-delay term is involved in (2), and therefore the model studied in this paper is more general than the corresponding ones in [33].

For convenience, in the following, for $r_{k}=i$, a matrix $M\left(r_{k}\right)$ will be denoted by $M_{i}$. For example, $A\left(r_{k}\right)$ is denoted by $A_{i}$.

When the sensors experience failures, we consider the following sensor failure model to describe the measured signal sent from sensors:

$$
y^{F}(k)=G y(k)
$$

where the sensor fault matrix $G$ is defined as follows:

$$
0 \leq \underline{G}=\operatorname{diag}\left\{\underline{g}_{1}, \ldots, \underline{g}_{p}\right\} \leq G=\operatorname{diag}\left\{g_{1}, \ldots, g_{p}\right\} \leq \bar{G}=\operatorname{diag}\left\{\bar{g}_{1}, \ldots, \bar{g}_{p}\right\} \leq I
$$

in which the variables $g_{i}(i=1, \ldots, p)$ quantify the failures of the sensors.

Letting

$$
\begin{gathered}
G_{0}=\operatorname{diag}\left\{g_{01}, \ldots, g_{0 p}\right\}:=\frac{\underline{G}+\bar{G}}{2}=\operatorname{diag}\left\{\frac{\underline{g}_{1}+\bar{g}_{1}}{2}, \ldots, \frac{\underline{g}_{p}+\bar{g}_{p}}{2}\right\}, \\
\tilde{G}=\operatorname{diag}\left\{\tilde{g}_{1}, \ldots, \tilde{g}_{p}\right\}:=\frac{\bar{G}-\underline{G}}{2}=\operatorname{diag}\left\{\frac{\bar{g}_{1}-\underline{g}_{1}}{2}, \ldots, \frac{\bar{g}_{p}-\underline{g}_{p}}{2}\right\},
\end{gathered}
$$

we can rewrite $G$ as follows:

$$
G=G_{0}+\Delta=G_{0}+\operatorname{diag}\left\{\phi_{1}, \ldots, \phi_{p}\right\}, \quad\left|\phi_{i}\right| \leq \tilde{g}_{i}, \quad(i=1, \ldots, p)
$$

In this paper, we consider the following reliable filter:

$$
\begin{aligned}
\hat{x}(k+1) & =A_{f}\left(r_{k}\right) \hat{x}(k)+B_{f}\left(r_{k}\right) y^{F}(k), \\
\hat{z}(k) & =C_{f}\left(r_{k}\right) \hat{x}(k)
\end{aligned}
$$

where $A_{f}\left(r_{k}\right), B_{f}\left(r_{k}\right)$ and $C_{f}\left(r_{k}\right)$ are parameters to be determined. By defining $\eta(k)=\left[\begin{array}{ll}x^{T}(k) & \hat{x}^{T}(k)\end{array}\right]^{T}$, we have the following filtering error system:

$$
\begin{aligned}
\eta(k+1) & =\tilde{A}\left(r_{k}\right) \eta(k)+\tilde{A}_{d}\left(r_{k}\right) \eta(k-d(k))+\tilde{B}\left(r_{k}\right) w(k)+Z^{T} f\left(Z \eta(k), Z \eta(k-d(k)), r_{k}\right), \\
e(k) & =\tilde{C}\left(r_{k}\right) \eta(k)
\end{aligned}
$$

where $e(k)=z(k)-\hat{z}(k)$ is the estimated error, and

$$
\begin{aligned}
& \tilde{A}\left(r_{k}\right)=\left[\begin{array}{cc}
A\left(r_{k}\right) & 0 \\
B_{f}\left(r_{k}\right) G C_{1}\left(r_{k}\right) & A_{f}\left(r_{k}\right)
\end{array}\right], \quad \tilde{A}_{d}\left(r_{k}\right)=\left[\begin{array}{cc}
A_{d}\left(r_{k}\right) & 0 \\
0 & 0
\end{array}\right], \\
& \tilde{B}\left(r_{k}\right)=\left[\begin{array}{c}
D\left(r_{k}\right) \\
B_{f}\left(r_{k}\right) G D_{1}\left(r_{k}\right)
\end{array}\right], \quad \tilde{C}\left(r_{k}\right)=\left[\begin{array}{ll}
C\left(r_{k}\right) & -C_{f}\left(r_{k}\right)
\end{array}\right], \quad Z=\left[\begin{array}{ll}
I & 0
\end{array}\right] .
\end{aligned}
$$


In this paper, we aim to design a reliable filter with the form (8) such that, for all admissible sensor failures, mode-dependent nonlinearity, time-varying delay and exogenous disturbance, the filtering error system (9) satisfies the following requirements:

(a) The filtering error system (9) is asymptotically mean-square stable.

(b) Under the zero-initial condition, the estimated error $e(k)$ satisfies

$$
\sum_{k=0}^{\infty} \mathbb{E}\left\{\|e(k)\|^{2}\right\}<\gamma^{2} \sum_{k=0}^{\infty} \mathbb{E}\left\{\|w(k)\|^{2}\right\}
$$

for all nonzero $w(k)$, where $\gamma>0$ is a prescribed scalar.

\section{MAIN REsults}

The following lemma is needed in the proofs of our main results in this paper.

Lemma 1: [24] Let $x \in \mathbb{R}^{n}$ and $y \in \mathbb{R}^{n}$. Then, for any scalar $\sigma>0$, we have

$$
x^{T} y+y^{T} x \leq \sigma x^{T} x+\sigma^{-1} y^{T} y .
$$

First of all, let us deal with both the stability analysis issue and the $H_{\infty}$ performance analysis issue in the case that the transition probabilities of the jumping process are completely known and the parameter matrix describing the sensor failures is known. Sufficient conditions for the addressed problem are obtained by using the semi-definite programme method.

Theorem 1: Consider the filtering error system (9) with known sensor failure parameter matrix $G$, completely known transition probabilities and a prescribed $H_{\infty}$ performance index $\gamma>0$. If there exist matrices $P_{i}>0, Q_{1}>0, Q_{2}>0, Q_{3}>0, S_{1}>0, S_{2}>0, R_{1}, R_{2}, R_{3}$ and scalars $\varepsilon_{l}>0(l=1, \ldots, q)$ such that the following linear matrix inequalities hold for any $i \in S$,

$$
\begin{aligned}
& {\left[\begin{array}{cccc}
-\varepsilon_{l} & * & * & * \\
\sum_{j \in S} \pi_{i j} P_{j} Z^{T} \theta_{l i} & -\sum_{j \in S} \pi_{i j} P_{j} & * & * \\
\lambda_{1} S_{1} Z^{T} \theta_{l i} & 0 & -S_{1} & * \\
\lambda_{2} S_{2} Z^{T} \theta_{l i} & 0 & 0 & -S_{2}
\end{array}\right]<0, \quad l=1, \ldots, q} \\
& {\left[\begin{array}{cccc}
\Omega_{i} & * & * & * \\
\lambda_{1} R_{1}^{T} & -S_{1} & * & * \\
\lambda_{2} R_{t}^{T} & 0 & -S_{2} & * \\
\Omega_{i}^{\prime} & 0 & 0 & \Omega_{i}^{\prime \prime}
\end{array}\right]<0, \quad t=2,3}
\end{aligned}
$$

where

$$
\begin{aligned}
& \Omega_{i}=-W_{P_{2}}^{T} P_{i} W_{P_{2}}+W_{Q_{1}}^{T} \bar{Q}_{1} W_{Q_{1}}+W_{Q_{2}}^{T} \bar{Q}_{2} W_{Q_{2}}+W_{Q_{3}}^{T} \bar{Q}_{3} W_{Q_{3}}-\gamma^{2} W_{w}^{T} W_{w} \\
& +R_{1} W_{R_{1}}+\left(R_{1} W_{R_{1}}\right)^{T}+R_{2} W_{R_{2}}+\left(R_{2} W_{R_{2}}\right)^{T}+R_{3} W_{R_{3}}+\left(R_{3} W_{R_{3}}\right)^{T},
\end{aligned}
$$

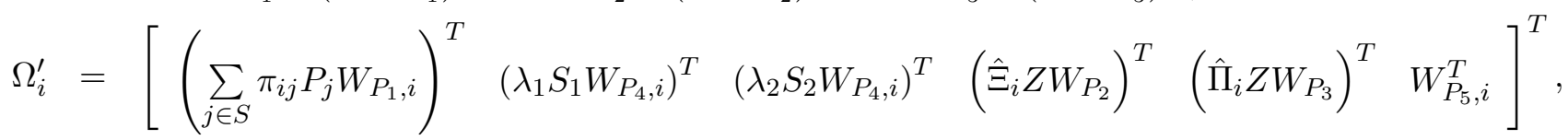

$$
\begin{aligned}
& \Omega_{i}^{\prime \prime}=\operatorname{diag}\left\{-\sum_{j \in S} \pi_{i j} P_{j},-S_{1},-S_{2},-E,-E,-I\right\}
\end{aligned}
$$




$$
\begin{aligned}
& \lambda_{1}=\sqrt{\tau}, \quad \lambda_{2}=\sqrt{d_{M}-\tau m}, \quad \lambda_{3}=\sqrt{d_{M}-\tau m+1}, \\
& \bar{Q}_{1}=\left[\begin{array}{cc}
Q_{1} & 0 \\
0 & -Q_{1}
\end{array}\right], \quad \bar{Q}_{2}=\left[\begin{array}{cc}
Q_{2} & 0 \\
0 & -Q_{2}
\end{array}\right], \quad \bar{Q}_{3}=\left[\begin{array}{cc}
Q_{3} & 0 \\
0 & -Q_{3}
\end{array}\right], \\
& W_{P_{1}, i}=\left[\begin{array}{lllll}
\tilde{A}_{i} & 0_{2 n, 2 m n} & \tilde{A}_{d i} & 0_{2 n} & \tilde{B}_{i}
\end{array}\right], \quad W_{P_{2}}=\left[\begin{array}{cc}
I_{2 n} & 0_{2 n, 2 m n+4 n+h}
\end{array}\right], \\
& W_{P_{3}}=\left[\begin{array}{lll}
0_{2 n, 2 m n+2 n} & I_{2 n} & 0_{2 n, 2 n+h}
\end{array}\right], \quad W_{P_{5}, i}=\left[\begin{array}{cc}
\tilde{C}_{i} & 0_{n_{C}, 2 m n+4 n+h}
\end{array}\right], \quad W_{P_{4}, i}=W_{P_{1}, i}-W_{P_{2}},
\end{aligned}
$$

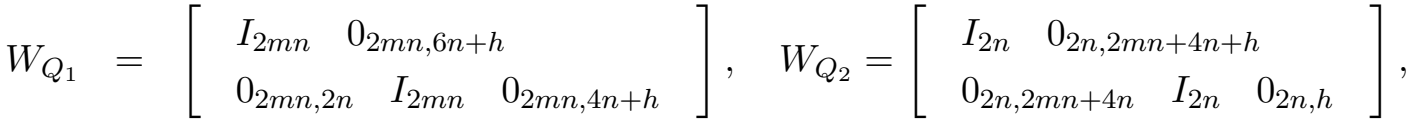

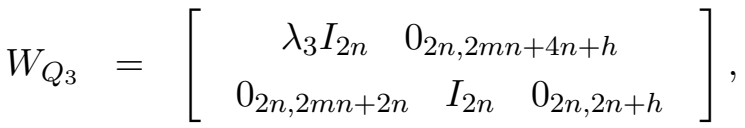

$$
\begin{aligned}
& W_{R_{1}}=\left[\begin{array}{lll}
I_{2 n} & -I_{2 n} & 0_{2 n, 2 m n+2 n+h}
\end{array}\right], \quad W_{R_{2}}=\left[\begin{array}{llll}
0_{2 n, 2 m n} & I_{2 n} & -I_{2 n} & 0_{2 n, 2 n+h}
\end{array}\right], \\
& W_{R_{3}}=\left[\begin{array}{llll}
0_{2 n, 2 m n+2 n} & I_{2 n} & -I_{2 n} & 0_{2 n, h}
\end{array}\right], \quad W_{w}=\left[\begin{array}{lll}
0_{h, 2 m n+6 n} & I_{h}
\end{array}\right], \\
& \hat{\Xi}_{i}=\left[\varepsilon_{1} \Xi_{1 i}^{\frac{1}{2}}, \ldots, \varepsilon_{l} \Xi_{l i}^{\frac{1}{2}}\right]^{T}, \quad \hat{\Pi}_{i}=\left[\varepsilon_{1} \Pi_{1 i}^{\frac{1}{2}}, \ldots, \varepsilon_{l} \Pi_{l i}^{\frac{1}{2}}\right]^{T}, \quad \mathrm{E}=\operatorname{diag}\left\{\varepsilon_{1} I, \ldots, \varepsilon_{l} I\right\}
\end{aligned}
$$

in which $n_{C}$ is the number of row in matrix $C_{i}$, then the filtering error system (9) is asymptotically mean-square stable with an $H_{\infty}$ disturbance attenuation level $\gamma$.

Proof: Let us first show that, under the zero-initial condition, the estimated error $e_{k}$ satisfies (10) for all nonzero $w_{k}$. Choose a new Lyapunov-Krasovskii functional candidate:

$$
V(k)=V_{1}(k)+V_{2}(k)+V_{3}(k)+V_{4}(k)
$$

where

$$
\begin{aligned}
& V_{1}(k)=\eta^{T}(k) P\left(r_{k}\right) \eta(k), \\
& V_{2}(k)=\sum_{\alpha=k-\tau}^{k-1} \Gamma^{T}(\alpha) Q_{1} \Gamma(\alpha)+\sum_{\alpha=k-d_{M}}^{k-1} \eta^{T}(\alpha) Q_{2} \eta(\alpha), \\
& V_{3}(k)=\sum_{\beta=-d_{M}+1} \sum_{\alpha=k-1+\beta}^{-\tau m+1} \eta^{T}(\alpha) Q_{3} \eta(\alpha), \\
& V_{4}(k)=\sum_{\beta=-\tau}^{-1} \sum_{\alpha=k+\beta}^{k-1} \delta^{T}(\alpha) S_{1} \delta(\alpha)+\sum_{\beta=-d_{M}}^{-\tau m-1} \sum_{\alpha=k+\beta}^{k-1} \delta^{T}(\alpha) S_{2} \delta(\alpha)
\end{aligned}
$$

with

$$
\delta(\alpha)=\eta(\alpha+1)-\eta(\alpha), \quad \Gamma(\alpha)=\left[\begin{array}{c}
\eta(\alpha) \\
\eta(\alpha-\tau) \\
\vdots \\
\eta(\alpha-(m-1) \tau)
\end{array}\right]
$$

For each $r_{k}=i \in S$, calculating the difference of $V(k)$ along the system (9) under the zero-initial condition, we have :

$$
\mathbb{E}\{\Delta V(k)\}=\mathbb{E}\left\{\Delta V_{1}(k)\right\}+\mathbb{E}\left\{\Delta V_{2}(k)\right\}+\mathbb{E}\left\{\Delta V_{3}(k)\right\}+\mathbb{E}\left\{\Delta V_{4}(k)\right\}
$$


where

$$
\begin{aligned}
& \mathbb{E}\left\{\Delta V_{1}(k)\right\}=\mathbb{E}\left\{\left[\tilde{A}_{i} \eta(k)+\tilde{A}_{d i} \eta(k-d(k))+\tilde{B}_{i} w(k)\right]^{T} \sum_{j \in S} \pi_{i j} P_{j}\right. \\
& \times\left[\tilde{A}_{i} \eta(k)+\tilde{A}_{d i} \eta(k-d(k))+\tilde{B}_{i} w(k)\right]-\eta^{T}(k) P_{i} \eta(k) \\
& \left.+\sum_{l=1}^{q}\left(\eta^{T}(k) Z^{T} \Xi_{l i} Z \eta(k)+\eta^{T}(k-d(k)) Z^{T} \Pi_{l i} Z \eta(k-d(k))\right) \operatorname{tr}\left(Z^{T} \theta_{l i} \theta_{l i}^{T} Z \sum_{j \in S} \pi_{i j} P_{j}\right)\right\} \\
& =\mathbb{E}\left\{\xi ^ { T } ( k ) \left(W_{P_{1}, i}^{T} \sum_{j \in S} \pi_{i j} P_{j} W_{P_{1}, i}+W_{P_{2}}^{T} \sum_{l=1}^{q} Z^{T} \Xi_{l i} Z \operatorname{tr}\left(Z^{T} \theta_{l i} \theta_{l i}^{T} Z \sum_{j \in S} \pi_{i j} P_{j}\right) W_{P_{2}}\right.\right. \\
& \left.\left.+W_{P_{3}}^{T} \sum_{l=1}^{q} Z^{T} \Pi_{l i} Z \operatorname{tr}\left(Z^{T} \theta_{l i} \theta_{l i}^{T} Z \sum_{j \in S} \pi_{i j} P_{j}\right) W_{P_{3}}-W_{P_{2}}^{T} P_{i} W_{P_{2}}\right) \xi(k)\right\}, \\
& \mathbb{E}\left\{\Delta V_{2}(k)\right\}=\mathbb{E}\left\{\Gamma^{T}(k) Q_{1} \Gamma(k)-\Gamma(k-\tau)^{T} Q_{1} \Gamma(k-\tau)+\eta(k)^{T} Q_{2} \eta(k)-\eta\left(k-d_{M}\right)^{T} Q_{2} \eta\left(k-d_{M}\right)\right\} \\
& =\mathbb{E}\left\{\xi^{T}(k)\left(W_{Q_{1}}^{T} \bar{Q}_{1} W_{Q_{1}}+W_{Q_{2}}^{T} \bar{Q}_{2} W_{Q_{2}}\right) \xi(k)\right\}, \\
& \mathbb{E}\left\{\Delta V_{3}(k)\right\}=\mathbb{E}\left\{\left(d_{M}-\tau m+1\right) \eta^{T}(k) Q_{3} \eta(k)-\sum_{\alpha=k-d_{M}}^{k-\tau m} \eta^{T}(\alpha) Q_{3} \eta(\alpha)\right\} \\
& \leq \mathbb{E}\left\{\left(d_{M}-\tau m+1\right) \eta^{T}(k) Q_{3} \eta(k)-\eta^{T}(k-d(k)) Q_{3} \eta(k-d(k))\right\} \\
& =\mathbb{E}\left\{\xi^{T}(k)\left(W_{Q_{3}}^{T} \bar{Q}_{3} W_{Q_{3}}\right) \xi(k)\right\}, \\
& \mathbb{E}\left\{\Delta V_{4}(k)\right\}=\mathbb{E}\left\{\delta^{T}(k)\left(\tau S_{1}+\left(d_{M}-\tau m\right) S_{2}\right) \delta(k)-\sum_{\alpha=k-\tau}^{k-1} \delta^{T}(\alpha) S_{1} \delta(\alpha)\right. \\
& \left.-\sum_{\alpha=k-d(k)}^{k-\tau m-1} \delta^{T}(\alpha) S_{2} \delta(\alpha)-\sum_{\alpha=k-d_{M}}^{k-d(k)-1} \delta^{T}(\alpha) S_{2} \delta(\alpha)\right\} \\
& =\mathbb{E}\left\{\left[\tilde{A}_{i} \eta(k)+\tilde{A}_{d i} \eta(k-d(k))+\tilde{B}_{i} w(k)-\eta(k)\right]^{T}\left(\tau S_{1}+\left(d_{M}-\tau m\right) S_{2}\right)\right. \\
& \times\left[\tilde{A}_{i} \eta(k)+\tilde{A}_{d i} \eta(k-d(k))+\tilde{B}_{i} w(k)-\eta(k)\right] \\
& +\sum_{l=1}^{q}\left(\eta^{T}(k) Z^{T} \Xi_{l i} Z \eta(k)+\eta^{T}(k-d(k)) Z^{T} \Pi_{l i} Z \eta(k-d(k))\right) \\
& \times \operatorname{tr}\left(Z^{T} \theta_{l i} \theta_{l i}^{T} Z\left(\tau S_{1}+\left(d_{M}-\tau m\right) S_{2}\right)\right) \\
& \left.-\sum_{\alpha=k-\tau}^{k-1} \delta^{T}(\alpha) S_{1} \delta(\alpha)-\sum_{\alpha=k-d(k)}^{k-\tau m-1} \delta^{T}(\alpha) S_{2} \delta(\alpha)-\sum_{\alpha=k-d_{M}}^{k-d(k)-1} \delta^{T}(\alpha) S_{2} \delta(\alpha)\right\}
\end{aligned}
$$




$$
\begin{aligned}
= & \mathbb{E}\left\{\xi ^ { T } ( k ) \left(\tau W_{P_{4}, i}^{T} S_{1} W_{P_{4}, i}+\left(d_{M}-\tau m\right) W_{P_{4}, i}^{T} S_{2} W_{P_{4}, i}\right.\right. \\
& +W_{P_{2}}^{T} \sum_{l=1}^{q} Z^{T} \Xi_{l i} Z \operatorname{tr}\left(Z^{T} \theta_{l i} \theta_{l i}^{T} Z\left(\tau S_{1}+\left(d_{M}-\tau m\right) S_{2}\right)\right) W_{P_{2}} \\
& \left.+W_{P_{3}}^{T} \sum_{l=1}^{q} Z^{T} \Pi_{l i} Z \operatorname{tr}\left(Z^{T} \theta_{l i} \theta_{l i}^{T} Z\left(\tau S_{1}+\left(d_{M}-\tau m\right) S_{2}\right)\right) W_{P_{3}}\right) \xi(k) \\
& \left.-\sum_{\alpha=k-\tau}^{k-1} \delta^{T}(\alpha) S_{1} \delta(\alpha)-\sum_{\alpha=k-d(k)}^{k-\tau m-1} \delta^{T}(\alpha) S_{2} \delta(\alpha)-\sum_{\alpha=k-d_{M}}^{k-d(k)-1} \delta^{T}(\alpha) S_{2} \delta(\alpha)\right\},
\end{aligned}
$$

with

$$
\xi(k)=\left[\begin{array}{lllll}
\Gamma^{T}(k) & \eta^{T}(k-\tau m) & \eta^{T}(k-d(k)) & \eta^{T}\left(k-d_{M}\right) & w^{T}(k)
\end{array}\right]^{T} .
$$

According to the definition of $\delta(\alpha)$, for any matrices $R_{1}, R_{2}$ and $R_{3}$, the following equations always hold

$$
\begin{aligned}
& 2 \xi(k)^{T} R_{1}\left[\eta(k)-\eta(k-\tau)-\sum_{\alpha=k-\tau}^{k-1} \delta(\alpha)\right]=0, \\
& 2 \xi(k)^{T} R_{2}\left[\eta(k-\tau m)-\eta(k-d(k))-\sum_{\alpha=k-d(k)}^{k-\tau m-1} \delta(\alpha)\right]=0 \\
& 2 \xi(k)^{T} R_{3}\left[\eta(k-d(k))-\eta\left(k-d_{M}\right)-\sum_{\alpha=k-d_{M}}^{k-d(k)-1} \delta(\alpha)\right]=0 .
\end{aligned}
$$

From (12), it is easy to get that

$$
\operatorname{tr}\left(Z^{T} \theta_{l i} \theta_{l i}^{T} Z\left(\sum_{j \in S} \pi_{i j} P_{j}+\tau S_{1}+\left(d_{M}-\tau m\right) S_{2}\right)\right)<\varepsilon_{l}(l=1, \ldots, q) .
$$

To analyze the $H_{\infty}$ performance of the filtering error system (9), we introduce the following index:

$$
\begin{aligned}
J(e, w) & =\sum_{k=0}^{\infty} \mathbb{E}\left\{e^{T}(k) e(k)-\gamma^{2} w^{T}(k) w(k)\right\} \\
& =\sum_{k=0}^{\infty} \mathbb{E}\left\{e^{T}(k) e(k)-\gamma^{2} w^{T}(k) w(k)+\Delta V(k)\right\}+\mathbb{E}\left\{V_{0}\right\}-\mathbb{E}\left\{V_{\infty}\right\} \\
& \leq \sum_{k=0}^{\infty} \mathbb{E}\left\{e^{T}(k) e(k)-\gamma^{2} w^{T}(k) w(k)+\Delta V(k)\right\} .
\end{aligned}
$$


From (15)-(23), we have

$$
\begin{aligned}
& \mathbb{E}\left\{e^{T}(k) e(k)-\gamma^{2} w^{T}(k) w(k)+\Delta V(k)\right\} \\
& \leq \mathbb{E}\left\{\xi ^ { T } ( k ) \left\{\Omega_{i}+W_{P_{1}, i}^{T} \sum_{j \in S} \pi_{i j} P_{j} W_{P_{1}, i}+\tau W_{P_{4}, i}^{T} S_{1} W_{P_{4}, i}+\left(d_{M}-\tau m\right) W_{P_{4}, i}^{T} S_{2} W_{P_{4}, i}+W_{P_{5}, i}^{T} W_{P_{5}, i}\right.\right. \\
& +W_{P_{2}}^{T} \sum_{l=1}^{q} Z^{T} \Xi_{l i} Z \operatorname{tr}\left(Z^{T} \theta_{l i} \theta_{l i}^{T} Z\left(\sum_{j \in S} \pi_{i j} P_{j}+\tau S_{1}+\left(d_{M}-\tau m\right) S_{2}\right)\right) W_{P_{2}} \\
& +W_{P_{3}}^{T} \sum_{l=1}^{q} Z^{T} \Pi_{l i} Z \operatorname{tr}\left(Z^{T} \theta_{l i} \theta_{l i}^{T} Z\left(\sum_{j \in S} \pi_{i j} P_{j}+\tau S_{1}+\left(d_{M}-\tau m\right) S_{2}\right)\right) W_{P_{3}} \\
& \left.\left.+\tau R_{1} S_{1}^{-1} R_{1}^{T}+(d(k)-\tau m) R_{2} S_{2}^{-1} R_{2}^{T}+\left(d_{M}-d(k)\right) R_{3} S_{2}^{-1} R_{3}^{T}\right\} \xi(k)\right\} \\
& -\sum_{\alpha=k-\tau}^{k-1}\left(S_{1} \delta(\alpha)+R_{1}^{T} \xi(k)\right)^{T} S_{1}^{-1}\left(S_{1} \delta(\alpha)+R_{1}^{T} \xi(k)\right) \\
& -\sum_{\alpha=k-d(k)}^{k-\tau m-1}\left(S_{2} \delta(\alpha)+R_{2}^{T} \xi(k)\right)^{T} S_{2}^{-1}\left(S_{2} \delta(\alpha)+R_{2}^{T} \xi(k)\right) \\
& -\sum_{\alpha=k-d_{M}}^{k-d(k)-1}\left(S_{2} \delta(\alpha)+R_{3}^{T} \xi(k)\right)^{T} S_{2}^{-1}\left(S_{2} \delta(\alpha)+R_{3}^{T} \xi(k)\right) \\
& \leq \mathbb{E}\left\{\xi ^ { T } ( k ) \left\{\Omega_{i}+W_{P_{1}, i}^{T} \sum_{j \in S} \pi_{i j} P_{j} W_{P_{1}, i}+\tau W_{P_{4}, i}^{T} S_{1} W_{P_{4}, i}+\left(d_{M}-\tau m\right) W_{P_{4}, i}^{T} S_{2} W_{P_{4}, i}+W_{P_{5}, i}^{T} W_{P_{5}, i}\right.\right. \\
& +W_{P_{2}}^{T} \sum_{l=1}^{q} \varepsilon_{l} Z^{T} \Xi_{l i} Z W_{P_{2}}+W_{P_{3}}^{T} \sum_{l=1}^{q} \varepsilon_{l} Z^{T} \Pi_{l i} Z W_{P_{3}} \\
& \left.\left.+\tau R_{1} S_{1}^{-1} R_{1}^{T}+(d(k)-\tau m) R_{2} S_{2}^{-1} R_{2}^{T}+\left(d_{M}-d(k)\right) R_{3} S_{2}^{-1} R_{3}^{T}\right\} \xi(k)\right\} \\
& =\mathbb{E}\left\{\xi ^ { T } ( k ) \left\{( \frac { d ( k ) - \tau m } { d _ { M } - \tau m } ) \left(\Omega_{i}+W_{P_{1}, i}^{T} \sum_{j \in S} \pi_{i j} P_{j} W_{P_{1}, i}+\tau W_{P_{4}, i}^{T} S_{1} W_{P_{4}, i}+\left(d_{M}-\tau m\right) W_{P_{4}, i}^{T} S_{2} W_{P_{4}, i}\right.\right.\right. \\
& \left.+W_{P_{5}, i}^{T} W_{P_{5}, i}+W_{P_{2}}^{T} \sum_{l=1}^{q} \varepsilon_{l} Z^{T} \Xi_{l i} Z W_{P_{2}}+W_{P_{3}}^{T} \sum_{l=1}^{q} \varepsilon_{l} Z^{T} \Pi_{l i} Z W_{P_{3}}+\tau R_{1} S_{1}^{-1} R_{1}^{T}+\left(d_{M}-\tau m\right) R_{2} S_{2}^{-1} R_{2}^{T}\right) \\
& +\left(\frac{d_{M}-d(k)}{d_{M}-\tau m}\right)\left(\Omega_{i}+W_{P_{1}, i}^{T} \sum_{j \in S} \pi_{i j} P_{j} W_{P_{1}, i}+\tau W_{P_{4}, i}^{T} S_{1} W_{P_{4}, i}+\left(d_{M}-\tau m\right) W_{P_{4}, i}^{T} S_{2} W_{P_{4}, i}+W_{P_{5}, i}^{T} W_{P_{5}, i}\right. \\
& \left.\left.\left.+W_{P_{2}}^{T} \sum_{l=1}^{q} \varepsilon_{l} Z^{T} \Xi_{l i} Z W_{P_{2}}+W_{P_{3}}^{T} \sum_{l=1}^{q} \varepsilon_{l} Z^{T} \Pi_{l i} Z W_{P_{3}}+\tau R_{1} S_{1}^{-1} R_{1}^{T}+\left(d_{M}-\tau m\right) R_{3} S_{2}^{-1} R_{3}^{T}\right)\right\} \xi(k)\right\} .
\end{aligned}
$$

By Schur complement, it follows from (13) that $\mathbb{E}\left\{e^{T}(k) e(k)-\gamma^{2} w^{T}(k) w(k)+\Delta V(k)\right\}<0$, which implies that $J(e, w)<0$. Therefore, the inequality (10) holds for all nonzero $w(k)$. Similar to the above deduction, we can show that the forward difference of $V(k)$ with $w=0$ satisfies $\Delta V(k)<0$, which indicates the filtering error system (9) is asymptotically mean-square stable. This completes the proof.

Remark 2: Based on the delay partitioning technique, asymptotically mean-square stability conditions of the filtering error system with a prescribed $H_{\infty}$ performance level have been obtained in Theorem 1 . The conditions can be checked by solving a set of LMIs. Note that the delay-fractioning approach has proven to be 
effective in reducing the possible conservatism but at the cost of added computation complexity. It is noticed that the dimensions of the LMIs depend on the partitioning number $m$. If $m$ increases, the dimensions of the LMIs will become larger and the computational burden will increase. Therefore, the partitioning number $m$ should be chosen properly.

Theorem 2: Consider the filtering error system (9) with known sensor failure parameter matrix $G$, completely known transition probabilities and a prescribed $H_{\infty}$ performance index $\gamma>0$. If there exist matrices $P_{i}>0, Q_{1}>0, Q_{2}>0, Q_{3}>0, S_{1}>0, S_{2}>0, R_{1}, R_{2}, R_{3}, H_{i}$ and scalars $\varepsilon_{l}>0(l=1, \ldots, q)$ such that the following linear matrix inequalities hold for any $i \in S$,

$$
\begin{aligned}
\Psi_{1 i} & =\left[\begin{array}{cccc}
-\varepsilon_{l} & * & * & * \\
H_{i}^{T} Z^{T} \theta_{l i} & H_{P, i} & * & * \\
\lambda_{1} H_{i}^{T} Z^{T} \theta_{l i} & 0 & H_{S_{1}, i} & * \\
\lambda_{2} H_{i}^{T} Z^{T} \theta_{l i} & 0 & 0 & H_{S_{2}, i}
\end{array}\right]<0, \quad l=1, \ldots, q \\
\Psi_{t i} & =\left[\begin{array}{cccc}
\Omega_{i} & * & * & * \\
\lambda_{1} R_{1}^{T} & -S_{1} & * & * \\
\lambda_{2} R_{t}^{T} & 0 & -S_{2} & * \\
\tilde{\Omega}_{i}^{\prime} & 0 & 0 & \tilde{\Omega}_{i}^{\prime \prime}
\end{array}\right]<0, \quad t=2,3
\end{aligned}
$$

where

$$
\begin{aligned}
& H_{P, i}=\sum_{j \in S} \pi_{i j} P_{j}-H_{i}-H_{i}^{T}, \quad H_{S_{1}, i}=S_{1}-H_{i}-H_{i}^{T}, \quad H_{S_{2}, i}=S_{2}-H_{i}-H_{i}^{T},
\end{aligned}
$$

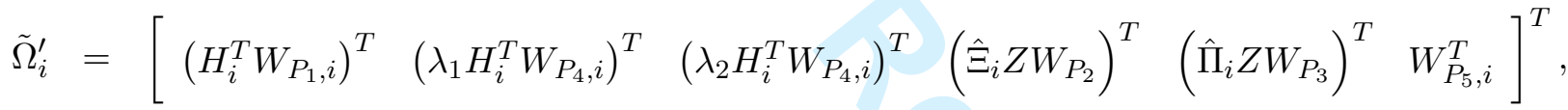

$$
\begin{aligned}
& \tilde{\Omega}_{i}^{\prime \prime}=\operatorname{diag}\left\{H_{P, i}, H_{S_{1}, i}, H_{S_{2}, i},-E,-E,-I\right\} \text {, }
\end{aligned}
$$

$\Omega_{i}, W_{P_{1}, i}, W_{P_{2}}, W_{P_{3}}, W_{P_{4}, i}, W_{P_{5}, i}, \lambda_{1}, \lambda_{2}$, E, $\hat{\Xi}_{i}$ and $\hat{\Pi}_{i}$ are defined as in Theorem 1, then the filtering error system (9) is asymptotically mean-square stable with an $H_{\infty}$ disturbance attenuation level $\gamma$.

Proof: Using the fact $\sum_{j \in S} \pi_{i j} P_{j}-H_{i}-H_{i}^{T} \geq-H_{i}^{T}\left(\sum_{j \in S} \pi_{i j} P_{j}\right)^{-1} H_{i}, S_{1}-H_{i}-H_{i}^{T} \geq-H_{i}^{T} S_{1}^{-1} H_{i}$ and $S_{2}-H_{i}-H_{i}^{T} \geq-H_{i}^{T} S_{2}^{-1} H_{i}$, we can obtain that

$$
\left[\begin{array}{cccc}
\Omega_{i} & * & * & * \\
\lambda_{1} R_{1}^{T} & -S_{1} & * & * \\
\lambda_{2} R_{t}^{T} & 0 & -S_{2} & * \\
\tilde{\Omega}_{i}^{\prime} & 0 & 0 & \tilde{\Omega}_{i}^{\prime \prime \prime}
\end{array}\right]<0, \quad t=2,3 .
$$

where

$$
\tilde{\Omega}_{i}^{\prime \prime \prime}=\operatorname{diag}\left\{-H_{i}^{T}\left(\sum_{j \in S} \pi_{i j} P_{j}\right)^{-1} H_{i},-H_{i}^{T} S_{1}^{-1} H_{i},-H_{i}^{T} S_{2}^{-1} H_{i},-E,-E,-I\right\} .
$$

Then, pre- and post-multiplying (28) by $\operatorname{diag}\left\{I, I, I, \sum_{j \in S} \pi_{i j} P_{j} H_{i}^{-T}, S_{1} H_{i}^{-T}, S_{2} H_{i}^{-T}, I, I, I\right\}$ and its transpose lead to (13). Similar to the above deduction, we can obtain that (26) can imply (12), respectively. Thus, the proof is completed. 
Remark 3: In Theorem 2, by introducing a variable $H$, the coupling between the Lyapunov matrices and the filtering error system matrices is eliminated. Such a newly introduced variable $H$ does not present any structural constraint such as symmetry, but is supposed to give potentially less conservative results.

In the case that the transition probabilities of the jumping process are partly unknown, the following theorem will present the conditions under which the filtering error system (9) is asymptotically mean-square stable with an $H_{\infty}$ disturbance attenuation level $\gamma$.

Theorem 3: Consider the filtering error system (9) with known sensor failure parameter matrix $G$, partly unknown transition probabilities and a prescribed $H_{\infty}$ performance index $\gamma>0$. If there exist matrices $P_{i}>0, Q_{1}>0, Q_{2}>0, Q_{3}>0, S_{1}>0, S_{2}>0, R_{1}, R_{2}, R_{3}, H_{i}$ and scalars $\varepsilon_{l}>0(l=1, \ldots, q)$ such that the following linear matrix inequalities hold for any $i \in S$,

$$
\begin{aligned}
\breve{\Psi}_{1 i}= & {\left[\begin{array}{cccc}
-\varepsilon_{l} & * & * & * \\
H_{i}^{T} Z^{T} \theta_{l i} & \breve{H}_{P, i} & * & * \\
\lambda_{1} H_{i}^{T} Z^{T} \theta_{l i} & 0 & H_{S_{1}, i} & * \\
\lambda_{2} H_{i}^{T} Z^{T} \theta_{l i} & 0 & 0 & H_{S_{2}, i}
\end{array}\right]<0, \quad l=1, \ldots, q } \\
\breve{\Psi}_{t i}= & {\left[\begin{array}{cccc}
\Omega_{i} & * & * & * \\
\lambda_{1} R_{1}^{T} & -S_{1} & * & * \\
\lambda_{2} R_{t}^{T} & 0 & -S_{2} & * \\
\tilde{\Omega}_{i}^{\prime} & 0 & 0 & \tilde{\Omega}_{i}^{\prime \prime \prime \prime}
\end{array}\right]<0, \quad t=2,3 }
\end{aligned}
$$

where

$$
\begin{aligned}
\breve{H}_{P, i} & =\Upsilon_{j}-H_{i}-H_{i}^{T}, \\
\tilde{\Omega}_{i}^{\prime \prime \prime \prime} & =\operatorname{diag}\left\{\breve{H}_{P, i}, H_{S_{1}, i}, H_{S_{2}, i},-E,-E,-I\right\},
\end{aligned}
$$

$\Omega_{i}, W_{P_{1}, i}, W_{P_{2}}, W_{P_{3}}, W_{P_{4}, i}, W_{P_{5}, i}, \lambda_{1}, \lambda_{2}, \mathrm{E}, \hat{\Xi}_{i}$ and $\hat{\Pi}_{i}$ are defined as in Theorem $1, \tilde{\Omega}_{i}^{\prime}, H_{S_{1}, i}$ and $H_{S_{2}, i}$ are defined as in Theorem 2 and if $\zeta_{\kappa}^{i}=\emptyset, \Upsilon_{j}=P_{j}$, otherwise,

$$
\left\{\begin{array}{l}
\Upsilon_{j}=\left(\sum_{j \in \zeta_{\kappa}^{i}} \pi_{i j}\right)^{-1} \sum_{j \in \zeta_{\kappa}^{i}} \pi_{i j} P_{j} \\
\Upsilon_{j}=P_{j}, \quad j \in \zeta_{u \kappa}^{i}
\end{array}\right.
$$

then the filtering error system (9) is asymptotically mean-square stable with an $H_{\infty}$ disturbance attenuation level $\gamma$.

Proof: For any $i \in S, \Psi_{t i}$ in $(27)$ can be rewritten as

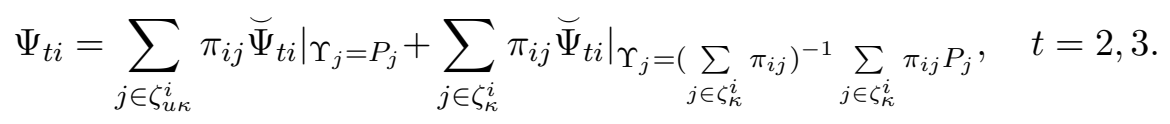

From (30), we can obtain that

$$
\begin{aligned}
& \left.\breve{\Psi}_{t i}\right|_{\Upsilon_{j}=P_{j}}<0, \quad j \in \zeta_{u \kappa}^{i}, \\
& \left.\breve{\Psi}_{t i}\right|_{\Upsilon_{j}=\left(\sum_{j \in \zeta_{\kappa}^{i}} \pi_{i j}\right)^{-1}} \sum_{j \in \zeta_{\kappa}^{i}} \pi_{i j} P_{j}
\end{aligned}
$$

Therefore, $\Psi_{t i}<0, t=2,3$. Similarly, we can have $\Psi_{1 i}<0$ from (29), respectively. Thus, the filtering error system (9) is asymptotically mean-square stable with partly unknown transition probabilities and an $H_{\infty}$ disturbance attenuation level $\gamma$. This completes the proof. 
Remark 4: In Theorem 3, the mean-square asymptotic stability conditions have been presented for the Markovian jump system (1) with partly unknown transition probabilities. If $\zeta_{u \kappa}^{i}=\emptyset, \forall i \in S$, the considered system is the one with completed known transition probabilities and the stability conditions in Theorem 3 are reduced to the ones in Theorem 2. If $\zeta_{\kappa}^{i}=\emptyset, \forall i \in S$, that is, all transition probabilities are unknown, it can be seen that Theorem 3 is still available. Judging from this, the developed results are much more general.

Based on Theorem 3, let us solve the problem of reliable $H_{\infty}$ filter design.

Theorem 4: Consider the filtering error system (9) with known sensor failure parameter matrix $G$, partly unknown transition probabilities and a prescribed $H_{\infty}$ performance index $\gamma>0$. If there exist matrices $P_{1 i}>0, P_{2 i}, P_{3 i}>0, Q_{1}>0, Q_{2}>0, Q_{3}>0, S_{11}>0, S_{12}, S_{13}>0, S_{21}>0, S_{22}, S_{23}>0, R_{1}, R_{2}, R_{3}, H_{1 i}$, $H_{2 i}, H_{3 i}, \hat{A}_{i}, \hat{B}_{i}, \hat{C}_{i}$ and scalars $\varepsilon_{l}>0(l=1, \ldots, q)$ such that the following linear matrix inequalities hold for any $i \in S$,

$$
\begin{aligned}
\hat{\Psi}_{1 i}= & {\left[\begin{array}{cccc}
-\varepsilon_{l} & * & * & * \\
\Phi_{4 i} & \Phi_{1 i} & * & * \\
\lambda_{1} \Phi_{4 i} & 0 & \Phi_{2 i} & * \\
\lambda_{2} \Phi_{4 i} & 0 & 0 & \Phi_{3 i}
\end{array}\right]<0, \quad l=1, \ldots, q } \\
\hat{\Psi}_{t i}= & {\left[\begin{array}{cccc}
\Phi_{7 i} & * & * & \\
\lambda_{1} R_{1}^{T} & -\Phi_{5} & * & * \\
\lambda_{2} R_{t}^{T} & 0 & -\Phi_{6} & * \\
\hat{\Omega}_{i}^{\prime} & 0 & 0 & \hat{\Omega}_{i}^{\prime \prime}
\end{array}\right]<0, \quad t=2,3 }
\end{aligned}
$$

where

$$
\begin{aligned}
& \Phi_{1 i}=\left[\begin{array}{cc}
\Upsilon_{1 j}-H_{1 i}-H_{1 i}^{T} & * \\
\Upsilon_{2 j}-H_{2 i}-H_{3 i}^{T} & \Upsilon_{3 j}-H_{2 i}-H_{2 i}^{T}
\end{array}\right], \quad \Phi_{2 i}=\left[\begin{array}{cc}
S_{11}-H_{1 i}-H_{1 i}^{T} & * \\
S_{12}-H_{2 i}-H_{3 i}^{T} & S_{13}-H_{2 i}-H_{2 i}^{T}
\end{array}\right], \\
& \Phi_{3 i}=\left[\begin{array}{cc}
S_{21}-H_{1 i}-H_{1 i}^{T} & * \\
S_{22}-H_{2 i}-H_{3 i}^{T} & S_{23}-H_{2 i}-H_{2 i}^{T}
\end{array}\right], \quad \Phi_{4 i}=\left[\begin{array}{c}
H_{1 i}^{T} \theta_{l i} \\
H_{3 i}^{T} \theta_{l i}
\end{array}\right], \\
& \Phi_{5}=\left[\begin{array}{cc}
S_{11} & * \\
S_{12} & S_{13}
\end{array}\right], \quad \Phi_{6}=\left[\begin{array}{cc}
S_{21} & * \\
S_{22} & S_{23}
\end{array}\right], \quad \Phi_{7 i}=\Omega_{i}, \quad P_{i}=\left[\begin{array}{cc}
P_{1 i} & * \\
P_{2 i} & P_{3 i}
\end{array}\right], \\
& \Phi_{8 i}=\left[\begin{array}{cccccc}
H_{1 i}^{T} A_{i}+\hat{B}_{i} G C_{1 i} & \hat{A}_{i} & 0_{n, 2 m n} & H_{1 i}^{T} A_{d i} & 0_{n, 3 n} & H_{1 i}^{T} D_{i}+\hat{B}_{i} G D_{1 i} \\
H_{3 i}^{T} A_{i}+\hat{B}_{i} G C_{1 i} & \hat{A}_{i} & 0_{n, 2 m n} & H_{3 i}^{T} A_{d i} & 0_{n, 3 n} & H_{3 i}^{T} D_{i}+\hat{B}_{i} G D_{1 i}
\end{array}\right], \\
& \Phi_{9 i}=\left[\begin{array}{cccccc}
H_{1 i}^{T} A_{i}-H_{1 i}^{T}+\hat{B}_{i} G C_{1 i} & \hat{A}_{i}-H_{2 i}^{T} & 0_{n, 2 m n} & H_{1 i}^{T} A_{d i} & 0_{n, 3 n} & H_{1 i}^{T} D_{i}+\hat{B}_{i} G D_{1 i} \\
H_{3 i}^{T} A_{i}-H_{3 i}^{T}+\hat{B}_{i} G C_{1 i} & \hat{A}_{i}-H_{2 i}^{T} & 0_{n, 2 m n} & H_{3 i}^{T} A_{d i} & 0_{n, 3 n} & H_{3 i}^{T} D_{i}+\hat{B}_{i} G D_{1 i}
\end{array}\right], \\
& \Phi_{10 i}=\left[\begin{array}{cc}
\varepsilon_{1} \Xi_{1 i}^{\frac{1}{2}} & 0_{n, 2 m n+5 n+h} \\
\vdots & \vdots \\
\varepsilon_{l} \Xi_{l i}^{\frac{1}{2}} & 0_{n, 2 m n+5 n+h}
\end{array}\right], \quad \Phi_{11 i}=\left[\begin{array}{ccc}
0_{n, 2 m n+2 n} & \varepsilon_{1} \Pi_{1 i}^{\frac{1}{2}} & 0_{n, 3 n+h} \\
\vdots & \vdots & \vdots \\
0_{n, 2 m n+2 n} & \varepsilon_{l} \Pi_{l i}^{\frac{1}{2}} & 0_{n, 3 n+h}
\end{array}\right] \text {, } \\
& \Phi_{12 i}=\left[\begin{array}{lll}
C_{i} & -\hat{C}_{i} & 0_{n_{C}, 2 m n+4 n+h}
\end{array}\right] \text {, } \\
& \hat{\Omega}_{i}^{\prime}=\left[\begin{array}{llllll}
\Phi_{8 i}^{T} & \lambda_{1} \Phi_{9 i}^{T} & \lambda_{2} \Phi_{9 i}^{T} & \Phi_{10 i}^{T} & \Phi_{11 i}^{T} & \Phi_{12 i}^{T}
\end{array}\right]^{T}, \quad \hat{\Omega}_{i}^{\prime \prime}=\operatorname{diag}\left\{\Phi_{1 i}, \Phi_{2 i}, \Phi_{3 i},-E,-E, I\right\},
\end{aligned}
$$


$\Omega_{i}$, E, $\lambda_{1}$ and $\lambda_{2}$ are defined as in Theorem 1, and if $\zeta_{\kappa}^{i}=\emptyset, \Upsilon_{1 j}=P_{1 j}, \Upsilon_{2 j}=P_{2 j}, \Upsilon_{3 j}=P_{3 j}$, otherwise,

$$
\begin{aligned}
& \left\{\begin{array}{l}
\Upsilon_{1 j}=\left(\sum_{j \in \zeta_{\kappa}^{i}} \pi_{i j}\right)^{-1} \sum_{j \in \zeta_{\kappa}^{i}} \pi_{i j} P_{1 j} \\
\Upsilon_{2 j}=\left(\sum_{j \in \zeta_{\kappa}^{i}} \pi_{i j}\right)^{-1} \sum_{j \in \zeta_{\kappa}^{i}} \pi_{i j} P_{2 j} \\
\Upsilon_{3 j}=\left(\sum_{j \in \zeta_{\kappa}^{i}} \pi_{i j}\right)^{-1} \sum_{j \in \zeta_{\kappa}^{i}} \pi_{i j} P_{3 j}
\end{array}\right. \\
& \left\{\begin{array}{l}
\Upsilon_{1 j}=P_{1 j} \\
\Upsilon_{2 j}=P_{2 j} \quad, \quad j \in \zeta_{u \kappa}^{i} \\
\Upsilon_{3 j}=P_{3 j}
\end{array}\right.
\end{aligned}
$$

then the filtering error system (9) is asymptotically mean-square stable with an $H_{\infty}$ disturbance attenuation level $\gamma$. Moreover, the parameters of the desired filter are given as follows:

$$
A_{f i}=H_{2 i}^{-T} \hat{A}_{i}, \quad B_{f i}=H_{2 i}^{-T} \hat{B}_{i}, \quad C_{f i}=\hat{C}_{i} .
$$

Proof: First, let us partition $H_{i}$ as

$$
H_{i}=\left[\begin{array}{ll}
H_{1 i} & H_{3 i} \\
H_{2 i} & H_{2 i}
\end{array}\right]
$$

where $H_{2 i}$ is nonsingular without loss of generality. Furthermore, partition $P_{i}, S_{1}$ and $S_{2}$ as

$$
P_{i}=\left[\begin{array}{cc}
P_{1 i} & * \\
P_{2 i} & P_{3 i}
\end{array}\right], \quad S_{1}=\left[\begin{array}{cc}
S_{11} & * \\
S_{12} & S_{13}
\end{array}\right], \quad S_{2}=\left[\begin{array}{cc}
S_{21} & * \\
S_{22} & S_{23}
\end{array}\right] .
$$

Substituting (35)-(37) into (29) and (30), we can get (33) and (34) immediately. This completes the proof.

In the following theorem, a design procedure for the desired filter parameters will be provided in the case that the failure parameter matrix is unknown but satisfies the constraints (4)-(7).

Theorem 5: Consider the filtering error system (9) with partly unknown transition probabilities and a prescribed $H_{\infty}$ performance index $\gamma>0$. If there exist matrices $P_{1 i}>0, P_{2 i}, P_{3 i}>0, Q_{1}>0, Q_{2}>0$, $Q_{3}>0, S_{11}>0, S_{12}, S_{13}>0, S_{21}>0, S_{22}, S_{23}>0, R_{1}, R_{2}, R_{3}, H_{1 i}, H_{2 i}, H_{3 i}, \hat{A}_{i}, \hat{B}_{i}, \hat{C}_{i}$ and scalars $\varepsilon_{l}>0(l=1, \ldots, q), \sigma>0$ such that the following linear matrix inequalities hold for any $i \in S$,

$$
\begin{aligned}
\tilde{\Psi}_{1 i}= & {\left[\begin{array}{cccc}
-\varepsilon_{l} & * & * & * \\
\Phi_{4 i} & \Phi_{1 i} & * & * \\
\lambda_{1} \Phi_{4 i} & 0 & \Phi_{2 i} & * \\
\lambda_{2} \Phi_{4 i} & 0 & 0 & \Phi_{3 i}
\end{array}\right]<0, \quad l=1, \ldots, q } \\
\tilde{\Psi}_{t i}= & {\left[\begin{array}{cccccc}
\Phi_{7 i} & * & * & * & * & * \\
\lambda_{1} R_{1}^{T} & -\Phi_{5} & * & * & * & * \\
\lambda_{2} R_{t}^{T} & 0 & -\Phi_{6} & * & * & * \\
\hat{\Omega}^{\prime \prime \prime} & 0 & 0 & \hat{\Omega}^{\prime \prime} & * & * \\
0 & 0 & 0 & \hat{\Omega}^{\prime \prime \prime \prime} & -\sigma \tilde{G}^{-2} & * \\
\sigma \Phi_{14 i} & 0 & 0 & 0 & 0 & -\sigma I
\end{array}\right]<0, \quad t=2,3 }
\end{aligned}
$$


where

$$
\begin{aligned}
& \tilde{\Phi}_{8 i}=\left[\begin{array}{cccccc}
H_{1 i}^{T} A_{i}+\hat{B}_{i} G_{0} C_{1 i} & \hat{A}_{i} & 0_{n, 2 m n} & H_{1 i}^{T} A_{d i} & 0_{n, 3 n} & H_{1 i}^{T} D_{i}+\hat{B}_{i} G_{0} D_{1 i} \\
H_{3 i}^{T} A_{i}+\hat{B}_{i} G_{0} C_{1 i} & \hat{A}_{i} & 0_{n, 2 m n} & H_{3 i}^{T} A_{d i} & 0_{n, 3 n} & H_{3 i}^{T} D_{i}+\hat{B}_{i} G_{0} D_{1 i}
\end{array}\right], \\
& \tilde{\Phi}_{9 i}=\left[\begin{array}{cccccc}
H_{1 i}^{T} A_{i}-H_{1 i}^{T}+\hat{B}_{i} G_{0} C_{1 i} & \hat{A}_{i}-H_{2 i}^{T} & 0_{n, 2 m n} & H_{1 i}^{T} A_{d i} & 0_{n, 3 n} & H_{1 i}^{T} D_{i}+\hat{B}_{i} G_{0} D_{1 i} \\
H_{3 i}^{T} A_{i}-H_{3 i}^{T}+\hat{B}_{i} G_{0} C_{1 i} & \hat{A}_{i}-H_{2 i}^{T} & 0_{n, 2 m n} & H_{3 i}^{T} A_{d i} & 0_{n, 3 n} & H_{3 i}^{T} D_{i}+\hat{B}_{i} G_{0} D_{1 i}
\end{array}\right], \\
& \Phi_{13 i}=\left[\begin{array}{cc}
\hat{B}_{i}^{T} & \hat{B}_{i}^{T}
\end{array}\right], \quad \Phi_{14 i}=\left[\begin{array}{lll}
C_{1 i} & 0_{n_{C_{1}}, 2 m n+5 n} & D_{1 i}
\end{array}\right], \\
& \hat{\Omega}_{i}^{\prime \prime \prime}=\left[\begin{array}{llllll}
\tilde{\Phi}_{8 i}^{T} & \lambda_{1} \tilde{\Phi}_{9 i}^{T} & \lambda_{2} \tilde{\Phi}_{9 i}^{T} & \Phi_{10 i}^{T} & \Phi_{11 i}^{T} & \Phi_{12 i}^{T}
\end{array}\right]^{T}, \\
& \hat{\Omega}_{i}^{\prime \prime \prime \prime}=\left[\begin{array}{llllll}
\Phi_{13 i} & \lambda_{1} \Phi_{13 i} & \lambda_{2} \Phi_{13 i} & 0 & 0 & 0
\end{array}\right] \text {, }
\end{aligned}
$$

$n_{C_{1}}$ is the number of row in matrix $C_{1 i}, \hat{\Omega}^{\prime \prime}, \Phi_{1 i}, \Phi_{2 i}, \Phi_{3 i}, \Phi_{4 i}, \Phi_{5}, \Phi_{6}, \Phi_{7 i}, \Phi_{10 i}, \Phi_{11 i}$ and $\Phi_{12 i}$ are defined as in Theorem 4, and E, $\lambda_{1}$ and $\lambda_{2}$ are defined as in Theorem 1, then the filtering error system (9) is asymptotically mean-square stable with an $H_{\infty}$ disturbance attenuation level $\gamma$. Moreover, the parameters of the desired filter are given as follows:

$$
A_{f i}=H_{2 i}^{-T} \hat{A}_{i}, \quad B_{f i}=H_{2 i}^{-T} \hat{B}_{i}, \quad C_{f i}=\hat{C}_{i} .
$$

Proof: From (7), we know that $\hat{\Psi}_{t i}$ in (34) can be rewritten as

$$
\hat{\Psi}_{t i}=\hat{\Psi}_{t i}^{\prime}+W^{T} \Delta V+V^{T} \Delta W
$$

where

$$
\begin{aligned}
\hat{\Psi}_{t i}^{\prime} & =\left[\begin{array}{cccc}
\Phi_{7 i} & * & * & * \\
\lambda_{1} R_{1}^{T} & -\Phi_{5} & * & * \\
\lambda_{2} R_{t}^{T} & 0 & -\Phi_{6} & * \\
\hat{\Omega}^{\prime \prime \prime} & 0 & 0 & \hat{\Omega}^{\prime \prime}
\end{array}\right]<0, \quad t=2,3 \\
W & =\left[\begin{array}{llll}
0 & 0 & 0 & \hat{\Omega}^{\prime \prime \prime \prime}
\end{array}\right], \quad V=\left[\begin{array}{llll}
\Phi_{14 i} & 0 & 0 & 0
\end{array}\right] .
\end{aligned}
$$

From Lemma 1 and (7), we have

$$
\hat{\Psi}_{t i} \leq \hat{\Psi}_{t i}^{\prime}+\sigma^{-1} W^{T} \tilde{G}^{2} W+\sigma V^{T} V=\Theta, \quad t=2,3 .
$$

By Schur complement, (39) implies that $\hat{\Psi}_{t i} \leq \Theta<0, t=2,3$. Therefore, the filtering error system (9) is asymptotically mean-square stable with partly unknown transition probabilities and an $H_{\infty}$ disturbance attenuation level $\gamma$. This completes the proof.

Remark 5: It is worth pointing out that the optimal $H_{\infty}$ performance index $\gamma^{*}$ depends on the number of known transition probabilities. Specifically, if more elements in the transition probability matrix can be obtained which are exactly known, a smaller index can be achieved for the optimal $H_{\infty}$ performance. This will be illustrated via a numerical example in the next section.

\section{An Illustrative Example}

In this section, we present a numerical example to demonstrate the effectiveness of the proposed method. 
Consider a discrete Markovian jump system of form (1) with four modes and the following parameters:

$$
\begin{aligned}
& A_{1}=\left[\begin{array}{cc}
0 & -0.2 \\
0.8 & 0.8
\end{array}\right], A_{2}=\left[\begin{array}{cc}
0 & -0.3 \\
0.8 & 0.9
\end{array}\right], A_{3}=\left[\begin{array}{cc}
0 & -0.2 \\
0.8 & 0.7
\end{array}\right], A_{4}=\left[\begin{array}{cc}
0 & -0.1 \\
0.8 & 0.6
\end{array}\right], \\
& A_{d 1}=A_{d 2}=A_{d 3}=A_{d 4}=\left[\begin{array}{cc}
0.1 & 0 \\
0 & 0.2
\end{array}\right], \quad C_{1}=C_{2}=C_{3}=C_{4}=\left[\begin{array}{ll}
0.1 & 0
\end{array}\right], \\
& D_{1}=D_{2}=D_{3}=D_{4}=\left[\begin{array}{c}
0.5 \\
0
\end{array}\right], \quad C_{11}=C_{12}=C_{13}=C_{14}=\left[\begin{array}{ll}
0.1 & 0.2
\end{array}\right], \\
& D_{11}=D_{12}=D_{13}=D_{14}=0.5, \quad \theta_{1}=\theta_{2}=\theta_{3}=\theta_{4}=\left[\begin{array}{c}
0.1 \\
0.1
\end{array}\right], \\
& \Xi_{1}=\Xi_{2}=\Xi_{3}=\Xi_{4}=\left[\begin{array}{cc}
0.01 & 0 \\
0 & 0.01
\end{array}\right], \quad \Pi_{1}=\Pi_{2}=\Pi_{3}=\Pi_{4}=\left[\begin{array}{cc}
0.01 & 0 \\
0 & 0.01
\end{array}\right] .
\end{aligned}
$$

The transition probability matrices for four different cases are given by

$$
\begin{aligned}
& \Lambda_{1}=\left[\begin{array}{cccc}
0.5 & 0.2 & 0.2 & 0.1 \\
0.4 & 0.2 & 0.3 & 0.1 \\
0.2 & 0.3 & 0.2 & 0.3 \\
0.2 & 0.2 & 0.2 & 0.4
\end{array}\right], \quad \Lambda_{2}=\left[\begin{array}{cccc}
0.5 & 0.2 & 0.2 & 0.1 \\
0.4 & ? & 0.3 & ? \\
0.2 & 0.3 & 0.2 & 0.3 \\
? & ? & 0.2 & 0.4
\end{array}\right] \text {, } \\
& \Lambda_{3}=\left[\begin{array}{cccc}
0.5 & 0.2 & 0.2 & 0.1 \\
0.4 & ? & 0.3 & ? \\
0.2 & ? & ? & ? \\
? & ? & 0.2 & 0.4
\end{array}\right], \quad \Lambda_{4}=\left[\begin{array}{cccc}
? & ? & ? & ? \\
? & ? & ? & ? \\
? & ? & ? & ? \\
? & ? & ? & ?
\end{array}\right]
\end{aligned}
$$

The time-varying delay $d_{k}$ satisfies $1 \leq d_{k} \leq 2$ and let $m=1$. The sensor fault matrix $G$ is assumed to satisfy $0.5 \leq G \leq 0.9$, and then we can obtain that $G_{0}=0.7$ and $\tilde{G}=0.2$.

Table I presents the optimal $H_{\infty}$ performance indices $\gamma^{*}$ for the four cases of different transition probabilities. From Table I, it can be seen that the more transition probabilities we know, the smaller optimal $H_{\infty}$ performance index the system can be achieved, which means that there exist tradeoffs between the cost of obtaining transition probabilities and the expectation of the system performance. On the other hand, for the system with the transition probability matrix $\Lambda_{3}$, by solving (39)-(38) in Theorem 5 , the desired filter parameters can be obtained as

$$
\begin{aligned}
& A_{f 1}=\left[\begin{array}{cc}
0.3311 & -0.0405 \\
0.3260 & 0.6109
\end{array}\right], \quad B_{f 1}=\left[\begin{array}{c}
0.2266 \\
-0.2894
\end{array}\right], \quad C_{f 1}=\left[\begin{array}{ll}
-0.0424 & 0.0115
\end{array}\right], \\
& A_{f 2}=\left[\begin{array}{cc}
0.2643 & -0.1517 \\
0.5572 & 0.7149
\end{array}\right], \quad B_{f 2}=\left[\begin{array}{c}
0.5826 \\
-0.8306
\end{array}\right], \quad C_{f 2}=\left[\begin{array}{ll}
-0.0815 & 0.0083
\end{array}\right] \text {, } \\
& A_{f 3}=\left[\begin{array}{cc}
0.2633 & -0.0711 \\
0.4487 & 0.4719
\end{array}\right], \quad B_{f 3}=\left[\begin{array}{c}
0.2161 \\
-0.1650
\end{array}\right], \quad C_{f 3}=\left[\begin{array}{ll}
-0.0465 & 0.0289
\end{array}\right], \\
& A_{f 4}=\left[\begin{array}{cc}
0.2404 & -0.0029 \\
0.4893 & 0.4544
\end{array}\right], \quad B_{f 4}=\left[\begin{array}{l}
0.0005 \\
0.1873
\end{array}\right], \quad C_{f 4}=\left[\begin{array}{ll}
-0.0531 & 0.0232
\end{array}\right] \text {. }
\end{aligned}
$$


TABLE I

MINIMUM $\gamma^{*}$ FOR DIFFERENT TRANSITION PROBABILITIES CASES

\begin{tabular}{ccccc}
\hline Transition probabilities & $\Lambda_{1}$ & $\Lambda_{2}$ & $\Lambda_{3}$ & $\Lambda_{4}$ \\
\hline$\gamma^{*}$ & 0.2398 & 0.2904 & 0.2911 & 0.2924 \\
\hline
\end{tabular}

\section{Conclusions}

In this paper, the reliable $H_{\infty}$ filtering problem has been investigated for a class of discrete-time nonlinear Markovian jump systems with sensor failures, time-varying delay and partly unknown transition probabilities. The systems under consideration are more general, which cover the Markovian jump systems with completely known and completely unknown transition probabilities as two special cases. A new Lyapunov-Krasovskii functional and delay-partitioning technique have been used to design a mode-dependent filter for all admissible uncertainties such that the filtering error system is asymptotically mean-square stable and achieves a prescribed $H_{\infty}$ performance level. The filter gains have been characterized by the solution of a set of LMIs. An illustrative example has been exploited to show the usefulness of the results obtained. The future research topics would include the extension of the main results developed in this paper to more general complex systems such as networked systems with random packet losses, general stochastic systems, polynomial nonlinear systems and functional differential equations of the neutral type.

\section{REFERENCES}

[1] S. Aberkane, J. C. Ponsart, M. Rodrigues and D. Sauter, Output feedback control of a class of stochastic hybrid systems, Automatica, vol. 44, no. 5, pp. 1325-1332, 2008.

[2] M. Basin, P. Shi and D. Calderon-Alvarez, Central suboptimal $H_{\infty}$ filter design for linear time-varying systems with state and measurement delays, Int. J. Systems Science, vol. 41, no. 4, pp. 411-421, 2010.

[3] M. Basin, M. A. Alcorta-Garcia and A. Alanis-Duran, Optimal filtering for linear systems with state and multiple observation delays, Int. J. Systems Science, vol. 39, no. 5, pp. 547-555, 2008.

[4] E. K. Boukas and Z. Liu, Robust $H_{\infty}$ control of discrete-time Markovian jump linear systems with mode-dependent timedelays, IEEE Transactions on Automatic Control, vol. 46, no. 12, pp. 1918-1924, 2001.

[5] A. Calzolari, P. Florchinger and G. Nappo, Nonlinear filtering for Markov systems with delayed observations, Int. J. Applied Mathematics and Computer Science, vol. 19, no. 1, pp. 49-57, 2009.

[6] Y. Cao and J. Lam, Robust $H_{\infty}$ control of uncertain Markovian jump systems with time-delay, IEEE Transactions on Automatic Control, vol. 45, no. 1, pp. 77-83, 2000.

[7] B. Chen, H. Li, P. Shi, C. Lin and Q. Zhou, Delay-dependent stability analysis and controller synthesis for Markovian jump systems with state and input delays, Information Sciences, vol. 179, no. 16, pp. 2851-2860, 2009.

[8] Z. Fei, H. Gao and P. Shi, New results on stabilization of Markovian jump systems with time delay, Automatica, vol. 45, no. 10, pp. 2300-2306, 2009.

[9] Y. Fujisaki and G. K. Befekadu, Reliable decentralised stabilisation of multi-channel systems: a design method via dilated LMIs and unknown disturbance observers, Int. J. Control, vol. 82, no. 11, pp. 2040-2050, 2009.

[10] D. P. Goodall and R. Postoyan, Output feedback stabilisation for uncertain nonlinear time-delay systems subject to input constraints, Int. J. Control, vol. 83, no. 4, pp. 676-693, 2010.

[11] D. P. Goodall, Stabilizing inputs for implicit functional differential equations of the neutral type with unknown time-varying delays, J. Dynamical and Control Systems, vol. 13, no. 2, pp. 177-215, 2007.

[12] Q. Han, A delay decomposition approach to stability of linear neutral systems, Proceeding of the 17th IFAC World Congress, pp. 2607-2612, Seoul, Korea, 2008.

[13] Y. He, Y. Zhang, M. Wu and J. She, Improved exponential stability for stochastic Markovian jump systems with nonlinearity and time-varying delay, Int. J. Robust and Nonlinear Control, vol. 20, no. 1, pp. 16-26, 2010.

[14] H. R. Karimi, Robust regulation with $H_{\infty}$ control of linear two-time-scale systems: a new modelling approach, Proc. Institution of Mechanical Engineering: Part I - J. Systems and Control Engineering, vol. 224, no. I3, pp. 235-246, 2010. 
[15] C. Lien, K. Yu, Y. Lin, Y. Chung and L. Chung, Robust reliable $H_{\infty}$ control for uncertain nonlinear systems via LMI approach, Applied Mathematics and Computation, vol. 198, no. 1, pp. 453-462, 2008.

[16] L. Liu, J. Wang and G. Yang, Reliable guaranteed variance filtering against sensor failure, IEEE Transactions on Signal Processing, vol. 51, no. 5, pp. 1403-1411, 2003.

[17] M. Liu, D. W. C. Ho and Y. Niu, Stabilization of Markovian jump linear system over networks with random communication delay, Automatica, vol. 45, no. 2, pp. 416-421, 2009.

[18] H. Ma and G. H. Yang, Fault-tolerant control synthesis for a class of nonlinear systems: Sum of squares optimization approach, Int. J. Robust and Nonlinear Control, vol. 19, no. 5, pp. 591-610, 2009.

[19] M. Staroswiecki and D. Berdjag, A general fault tolerant linear quadratic control strategy under actuator outages, Int. J. Systems Science, vol. 41, no. 8, pp. 971-985, 2010.

[20] A. Tellili, M. N. Abdelkrim and M. Benrejeb, Reliable $H_{\infty}$ control of multiple time scales singularly perturbed systems with sensor failure, Int. J. Control, vol. 80, no. 5, pp. 659-665, 2007.

[21] Z. Wang, J. Lam and X. Liu, Robust filtering for discrete-time Markovian jump delay systems, IEEE Signal Processing Letters, vol. 11, no. 8, pp. 659-662, 2004.

[22] Z. Wang, B. Huang and H. Unbehauen, Robust reliable control for a class of uncertain nonlinear state-delayed systems, Automatica, vol. 35, pp. 955-963, 1999.

[23] G. Wei, Z. Wang and H. Shu, Nonlinear $H_{\infty}$ control of stochastic time-delay systems with Markovian switching, Chaos, Solitons and Fractals, vol. 35, pp. 442-451, 2008.

[24] L. Xie, C. de Souza and M. Fu, $H_{\infty}$ estimation for discrete time linear uncertain systems, Int. J. Robust Nonlinear Contr., vol. 1, pp. 111-123, 1991.

[25] J. Xiong, J. Lam, H. Gao and D. W. C. Ho, On robust stabilization of Markovian jump systems with uncertain switching probabilities, Automatica, vol. 41, no. 5, pp. 897-903, 2005.

[26] G. H. Yang and D. Ye, Adaptive reliable $H_{\infty}$ filtering against sensor failures, IEEE Transactions on Signal Processing, vol. 55, no. 7, pp. 3161-3171, 2007.

[27] C. Yuan and X. Mao, Robust stability and controllability of stochastic differential delay equations with Markovian switching, Automatica, vol. 40, pp. 343-354, 2004.

[28] L. Zhang, E. K. Boukas, L. Baron, H. R. Karimi, Fault detection for discrete-time Markov jump linear systems with partially known transition probabilities, Int. J. Control, vol. 83, no. 8, pp. 1564-1572, 2010.

[29] L. Zhang and E. K. Boukas, Mode-dependent $H_{\infty}$ filtering for discrete-time Markovian jump linear systems with partly unknown transition probabilities, Automatica, vol. 45, no. 6, pp. 1462-1467, 2009.

[30] X. Zhang, G. Lu and Y. Zheng, Observer design for descriptor Markovian jumping systems with nonlinear perturbations, Circuits, Systems and Signal Processing, vol. 27, no. 1, pp. 95-112, 2008.

[31] Y. Zhao, H. Gao, J. Lam and B. Du, Stability and stabilization of delayed T-S fuzzy systems: a delay partitioning approach, IEEE Transactions on Fuzzy Systems, vol. 17, no. 4, pp. 750-762, 2009.

[32] W. Zhou, H. Lu, C. Duan and M. Li, Delay-dependent robust control for singular discrete-time Markovian jump systems with time-varying delay, Int. J. Robust and Nonlinear Control, vol. 20, no. 10, pp. 1112-1128, 2010.

[33] Y. I. Yaz and E. E. Yaz, On LMI formulations of some problems arising in nonlinear stochastic system analysis, IEEE Transactions on Automatic Control, vol. 44, no. 4, pp. 813-816, 1999.

[34] H. Gao, Y. Zhao, J. Lam, and K. Chen, $H_{\infty}$ fuzzy filtering of nonlinear systems with intermittent measurements, IEEE Transactions on Fuzzy Systems, vol. 17, no. 2, pp. 291-300, 2009.

[35] X. Meng, J. Lamb, B. Du and H. Gao, A delay-partitioning approach to the stability analysis of discrete-time systems, Automatica, vol. 46, pp. 610-614, 2010.

[36] Z. Wu, H. Su and J. Chu, $H_{\infty}$ filtering for singular systems with time-varying delay, International Journal of Robust and Nonlinear Control, vol. 20, no. 11, pp. 1269-1284, 2010.

[37] Z. Wu, H. Su and J. Chu, $H_{\infty}$ filtering for singular Markovian jump systems with time delay, International Journal of Robust and Nonlinear Control, vol. 20, no. 8, pp. 939-957, 2010. 